\title{
Energy coupling in short pulse laser solid interactions and its impact for space debris removal
}

\author{
David Neely, ${ }^{1,2^{*}}$ Ric Allott, ${ }^{1}$ Bob Bingham, ${ }^{1,}$, John Collier, ${ }^{1}$ Justin Greenhalgh, ${ }^{1}$ Max Michaelis ${ }^{3}$, \\ Jonathan Phillips ${ }^{1}$, Claude R. Phipps ${ }^{4}$ and Paul McKenna ${ }^{2}$ \\ ${ }^{1}$ Central Laser Facility, STFC, Rutherford Appleton Laboratory, Chilton, Didcot, Oxon, OX11 OQX, UK \\ ${ }^{2}$ Department of Physics, SUPA, University of Strathclyde, Glasgow, G4 ONG, UK \\ ${ }^{3}$ School of Chemistry and Physics, University of KwaZulu-Natal, South Africa \\ ${ }^{4}$ Photonic Associates, LLC, $200 A$ Ojo de la Vaca Road, Santa Fe NM 87508, USA, \\ *Corresponding author: author_david.neely@stfc.ac.uk
}

Received Month X, XXXX; revised Month X, XXXX; accepted Month X, XXXX; posted Month X, XXXX (Doc. ID XXXXX); published Month X, XXXX

\begin{abstract}
Significant advances have been made over the last decade to improve the performance, efficiency and contrast of high peak and average power laser systems, driven by their use in a wide variety of fields, from the industrial to the scientific. As the contrast of the lasers has improved, interactions with contrasts of $10^{12}$ are now routinely undertaken. At such high contrasts, there is negligible pre-plasma formation and the ionized surface layer created by sub ps duration pulses typically forms a highly reflective "plasma mirror" capable of reflecting between $70-90 \%$ of the incident energy. Although such interactions are of significant interest for applications such as harmonic source production and to enable the underlying physics to be studied, their low absorption can limit their usefulness for applications such as space debris removal.
\end{abstract}

OCIS codes: (140.3440) Laser-induced breakdown; (260.3230) Ionization; (260.7120) Ultrafast phenomena. http://dx.doi/org/

\section{Introduction}

The growing demand for utilization of satellites by governments, companies and individuals is increasingly threatened by collisions with debris in low earth orbit $(<2000 \mathrm{~km})$. The potential for a self-sustaining cascade of collisions known as the Kessler syndrome [1] is increasing as the number of satellites and pieces of space debris has risen over the last four decades. There is a growing realization [2-3] amongst the community capable of orbital launches, that all future satellites must have adequate maneuvering capability so that they can be placed in a mid-term (25 year) decay orbit after their intended operational life, or safely de-orbited [4] by other techniques [5].

A laser based system for de-orbiting either the space debris or satellites after the end of their life cycle was first proposed [6] almost 20 years ago. Although the recoil from photon reflection/absorption could be used to de-orbit an object, the momentum imparted by laser ablated material is typically $\sim 10^{4}$ times larger for a given quantity of photons, if the power density is sufficient to cause ablation. Therefore, most designs have considered a high power laser system either terrestrially based or in orbit which could be repeatedly focused onto an object causing material ablation and an orbital velocity change. Recent advances in laser technology over the last two decades have significantly increased the repetition rate and scope of available optical parameters which could be delivered. In the field of high intensity systems, the peak optical powers have typically increased from TW to PW's, with pulse lengths reducing from ns to 100 's fs. The laser pumping technology is also advancing with the introduction of Diode Pumped Solid State Lasers [7]. Here, the emission wavelength of the pumping source can be matched to the lasing material absorption to ensure efficient coupling and electrical to lasing efficiencies of 10-20\% are now deliverable.

In this paper, the role of the plasma density scale length present during the interaction of short sub ps pulses on laser energy absorption will be explored and the consequences for orbital debris removal discussed.

\section{Laser debris removal system requirements}

When an intense laser pulse illuminates a surface at a fluence $\Phi\left(\mathrm{J} / \mathrm{m}^{2}\right)$, it is possible to cause ablation if the fluence exceeds the threshold for that material $\Phi>\Phi_{\mathrm{t}}$. Optical ablation has been studied by many groups [8] and it was generally found that the fluence required for plasma ignition [9] $5 \times 10^{8} \tau^{0.5}$, where $\tau$ is the duration of the illuminating pulse (for $\tau>\mathrm{ps}$ ). The damage threshold measured at wavelength $\lambda=1.05 \times 10^{-6} \mathrm{~m}$ (the wavelength of $\mathrm{Nd}$-doped glass lasers) in the regime $100 \mathrm{~ns}>\tau>1$ ps for a number of materials is shown in Figure 1. It can be seen, that when $\tau>100$ ps the damage threshold is a vertical line on 
Figure 1 for a given material. In this regime of pulse lengths, damage is considered to be caused by energy absorption and transfer to the material's lattice. As thermal conduction dominates the heat flow, a $\tau^{0.5}$ dependence on $\Phi_{t}(\tau)$ is to be expected [10]. For pulses of a few ps and less, the energy is deposited into a surface layer for an absorbing metal and heating above the boiling point is sufficiently rapid that negligible heat conduction takes place. Hence, the line on Figure 1 becomes nearly horizontal for pulses < few ps. Intermediate between 1 and $100 \mathrm{ps}$, there is a changeover region for the heat flow and this can be observed as a smooth transition, the exact location of which is material dependent.

As well as being above the ablation threshold, the efficiency with which a given laser pulse generates pressure must be understood before a choice of suitable design parameters for an orbital debris removal system can be selected. We can use the momentum coupling parameter $\mathrm{C}_{m}$ which is the pressure $\mathrm{p}$ exerted on the target divided by the Intensity I $\left(\mathrm{Wm}^{-2}\right)$ [9]. Phipps [11] has correlated data from a number of studies at various wavelengths and pulse durations and the trend line for $\mathrm{C}_{\mathrm{m}}$ is plotted against $I \lambda \tau^{0.5}$ as shown in Figure 1 . If the product of $I \lambda \tau^{0.5}$ is too low $\left(<100 \mathrm{Jm}^{-1} \mathrm{~s}^{-0.5}\right)$, thermal conduction works to limit the temperature rise and no ablation occurs. As $I \lambda \tau^{0.5}$ is increased, significant surface heating occurs and the material may vaporize if $\Phi>\Phi_{\mathrm{t}}(\tau)$ causing $\mathrm{C}_{\mathrm{m}}$ to rapidly rise to a maximum. Initially, the laser can still penetrate through the transparent vapor and deposit energy directly into the original surface. However, there is a rapid transition to absorbing plasma and the laser now deposits energy above the surface, imparting force on the target through the plasma pressure. As the temperature of the plasma increases, a greater fraction of the incident energy is used to ionize the plasma and more and more energy is lost to escaping radiation, hence, $\mathrm{C}_{\mathrm{m}}$ slowly decreases. For ultra-high intensities $\left(\mathrm{I} \lambda \tau^{0.5} \gg>10^{9}\right)$ the light pressure can dominate over the plasma pressure and push the absorbing surface back towards solid densities. However, at such intensities most of the energy is transported away from the focus in relativistic electrons [12], which can deposit their energy far into the target and $\mathrm{C}_{\mathrm{m}}$ is low.

The extreme temperatures and gradients associated with short pulse illumination can also cause the expulsion of a wide

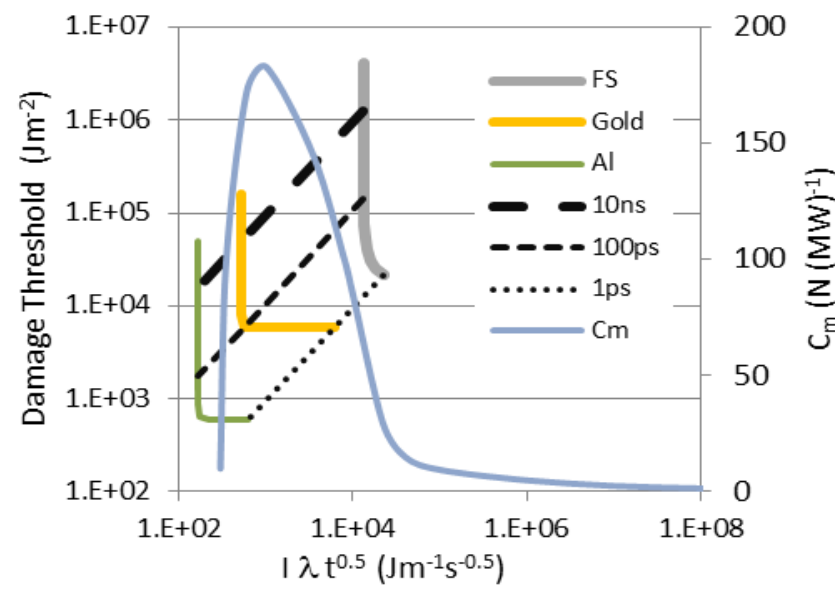

Figure 1. Damage threshold plotted as a function of $\mathrm{I} \lambda \tau^{0.5}$ for Fused silica, gold and aluminium, compiled from [8] measured with laser pulses of $1.05 \times 10^{-6} \mathrm{~m}$ wavelength. The intersection with the dashed lines shows the damage thresholds for $1 \mathrm{ps}, 100 \mathrm{ps}$ and $10 \mathrm{~ns}$ respectively. Also shown is the momentum coupling parameter from [9] for polymer and aluminium targets, which reads against the scale on the right. variety of states of matter from ions [13], atoms, clusters, nanoparticles [14] and droplets. Amoruso et al [14] found that at intensities a few times above threshold, three main components to the expanding plume were identified: a low number of fast ions at $\sim 10^{5} \mathrm{~ms}^{-1}$; a much larger neutral and ion population at $\sim 10^{4}$ $\mathrm{ms}^{-1}$; and a much slower component at $10^{2} \mathrm{~ms}^{-1}$ containing 10 's $\mathrm{nm}$ sized nanoparticles. Using a simple model based on assuming that the dominant absorption mechanism was due to avalanche ionization, good estimates for the ablation thresholds were obtained in the sub-ps duration interaction regime. However, it is only the trailing edge of the pulse that is strongly absorbed and Amoruso also noted that strong reflection could occur.

\section{Plasmas as reflectors}

When studying the absorption of short pulse ( ps) intense lasers with solid matter, it has long been realized that the presence of a pre-plasma can significantly change the interaction physics [15-16]. Although the absorbed energy $\mathrm{E}_{\mathrm{a}}$ can be determined from measurements made using an integrating sphere, in which all the scattered, transmitted [17] or reflected laser energy is collected and subtracted from the incident energy $\mathrm{E}$, this is intrusive as it requires a large solid angle, blocking any other diagnostic measurements. A more common technique implemented on many experiments is to measure the specularly reflected laser energy $\mathrm{E}_{\text {spec }}$ and assume $\mathrm{E}_{\mathrm{a}}=\mathrm{E}-\mathrm{E}_{\text {spec }}$ in the absence of significant scatter or transmission. For medium-to-high contrast laser systems, where the intensity contrast is typically $>$ $10^{6}[18]$ and can be up to $10^{12}$ a few ps before the arrival of the main pulse, it has been found that the ionized surface can act as a very efficient [19] almost optically flat "plasma mirror" during the interaction [20]. The specular reflectivity of fused silica measured by Ziener [19] for 90 and 500 fs duration pulses at 800 $\mathrm{nm}$ is shown in figure 2 . As expected, the switch-on in reflectivity for the shorter pulse $90 \mathrm{fs}$ data is at a slightly higher of $I \lambda \tau^{0.5}$ than the 500 fs data due to the scaling of $\Phi_{\mathrm{t}}(\tau)$ for shorter pulses [8]. As the intensity is increased above threshold, the reflectivity increases and peaks at $\sim 70 \%$ for $10^{7}<\mathrm{I} \lambda \tau^{0.5}<10^{8} \mathrm{Jm}^{-1} \mathrm{~s}^{-0.5}$ and then drops rapidly for $\mathrm{I} \mathrm{t}^{0.5}>10^{9} \mathrm{Jm}^{-1} \mathrm{~s}^{-0.5}$. This fall-off in reflectivity is associated with the plasma being sufficiently hot that it can significantly expand hydro-dynamically during the period of illumination. Also shown on Figure 2. is the optical transmission

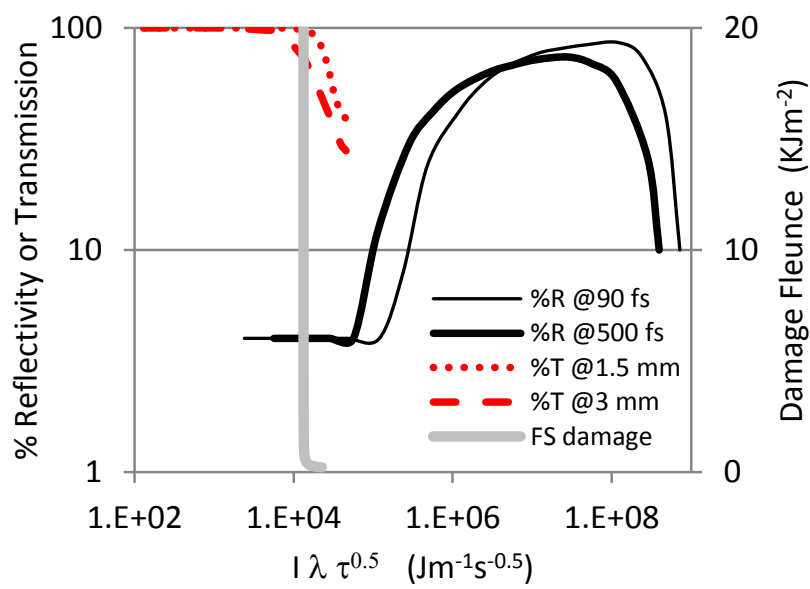

Figure 2. The specular reflectivity for Fused Silica using 90 and 500 fs, $8 \times 10^{-7} \mathrm{~m}$ pulsed illumination adapted from [19] is plotted as a function of $\mathrm{I} \lambda \tau^{0.5}$. The transmission (red lines) through a 1.5 and $3 \mathrm{~mm}$ thick fused silica sample is also displayed showing good alignment with the damage threshold curve shown in grey. 
of fused silica measured for 1.5 and $3 \mathrm{~mm}$ samples. The transmitted energy through the $3 \mathrm{~mm}$ sample shows a lower threshold value and lower transmission (once above threshold). This effect is associated with self-focusing as the beam passes through the sample which can cause filamentation down to diameters on the order of $\lambda$ and locally raises the fluence above $\Phi_{\mathrm{t}}(\tau)$ causing energy deposition within the bulk of the media.

Experiments have also been carried out where the level of laser energy preceding the main $50 \mathrm{fs}$ pulse has been varied and the specular reflectivity measured [21]. Although these measurements were carried out for rather high values of $I \lambda t^{0.5}>$ $10^{8}$ they clearly demonstrate, that a larger pre-plasma present before the main pulse can significantly reduce the plasma reflectivity and hence potentially increase absorption. However, at values of $I \lambda t^{0.5}$ closer to the peak for momentum coupling, the energy required to generate a pre-plasma with large scale length could represent a significant fraction of the energy contained in the main peak. Work has been carried out using such fs/ns pulse combinations [22, 23] for ablation studies and it was generally found to be advantageous. However, specific experiments would need to be undertaken to find if a more efficient regime for impulse generation can be found.

The reflectivity for a metal, in this case gold, measured for a 0.5 ps pulse of wavelength $1.054 \times 10^{-6} \mathrm{~m}$ from below the ablation threshold to $\mathrm{I} \lambda \tau^{0.5}=10^{8} \mathrm{Jm}^{-1} \mathrm{~S}^{-0.5}$ for $\mathrm{S}$ polarized laser light is shown in Figure 3 . This curve shows very high reflectivity $>90 \%$ for low values of $\mathrm{I} \lambda \tau^{0.5}<10^{5} \mathrm{Jm}^{-1} \mathrm{~S}^{-0.5}$ leaving the maximum energy deposited into the plasma to be $<10 \%$. Therefore, there will be very low coupling efficiency into a plasma. This should be contrasted against the case of using long $(\sim n s)$ pulses where the absorption is dominated by Inverse Bremsstrahlung heating and absorption fractions into the plasma of $>25 \%$ are common. The reflectivity for the short pulse case only drops significantly for very high values of $\mathrm{I} \lambda \tau^{0.5}>10^{8} \mathrm{Jm}^{-1} \mathrm{~s}^{-0.5}$. This would again be unsuitable for generating efficient momentum.

\section{Conclusion}

Short duration illumination $(\leq 1 \mathrm{ps})$ where there is limited heat conduction into the target compared to the ns regime was considered to be advantageous for both laser propulsion and debris removal [11] in the mid 1990's. Recent measurements demonstrate that, for pre-pulse-free laser interactions with solid matter above the ablation threshold, the majority of energy is reflected rather than being absorbed. This is found to be generic across many systems where the pulse length is in the $50 \mathrm{fs}-\sim \mathrm{ps}$

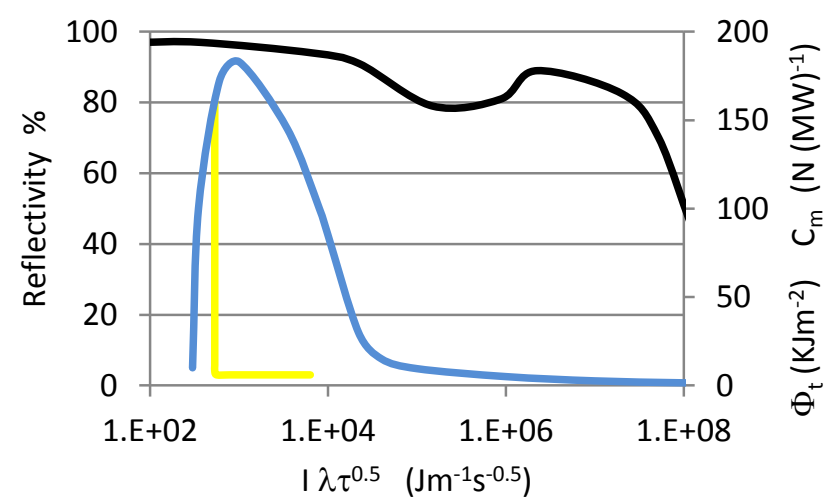

Figure 3. Graph showing the reflectivity for a solid gold target for ps illumination at s polarisation. Also shown is the general impulse coupling parameter and the yellow line indicates the fluence threshold for damage. regime and the intensities are within a few orders of magnitude above the ablation threshold $\Phi_{\mathrm{t}}$. For intensities many orders of magnitude above $\Phi_{\mathrm{t}}$, different absorption mechanisms can dominate. However, at $\mathrm{I} \lambda \tau^{0.5}>10^{8}$, for sub ps interactions, the electron and ion temperatures become significant and only a small fraction of the energy goes into producing momentum. New studies measuring the momentum coupling using ns, low contrast or combined ps/ns laser systems will help determine the optimum interaction conditions for orbital debris removal.

1 D. J. Kessler, "Orbital debris from upper-stage breakup" Progress in Astronautics and Aeronautics, vol. 121, p. 3, 1989.

$2 \mathrm{H}$. G. Lewis et al, "Synergy of debris mitigation and removal" Acta Astronautica, 81, p62-68, 2012

$3 \mathrm{~J}$. C. Liou et al, "Controlling the growth of future LEO debris populations with active debris removal" Acta Astronautica, 66, 5-6, p648-653, 2010

4 A. E. White and H. G. Lewis. "An adaptive strategy for active debris removal" Advances in Space Research, 53, 8, p1195-1206 2014.

5 C. Bonnal et al, "Active debris removal: Recent progress and current trends" Acta Astronautica, 85, p51-60, 2013

6 C. Phipps, H. Friedman et al. "ORION: clearing near-Earth space debris using a 20-kW, 530-nm Earth-based, repetitively pulsed laser" Laser Particle Beams 14, p1-44, 1996a.

$7 \mathrm{~K}$. Ertle et al, "Optimising the efficiency of pulsed diode pumped Yb:YAG laser amplifiers for ns pulse generation" Opt. Express, 19, 27, p26610-26626, 2011.

8 B. C. Stuart et al, "Optical ablation by high-power short-pulse lasers", J. opt. Soc. Am. B 13, 2, p459-468 1996.

$9 \mathrm{~J}$. E. Sinko, et al, "Modelling $\mathrm{CO}_{2}$ laser ablation impulse of polymers in vapour and plasma regimes" Appl. Phys. Lett 95, 131105, 2009

10 E. S. Bliss, "Pulse duration dependence of laser damage mechanisms" Electro-optics, 3, p99-108, 1971

11 C. R. Phipps, "Laser Interaction and related phenomena" AIP Conference Proceedings 406, p477-484, 1997

12 R. Gray et al, "Surface transport of energetic electrons in intense ps laser foil interactions" Appl. Phys. Lett 99, 17, 171502, 2011

$13 \mathrm{D}$. Neely et al, "Enhanced proton beams from ultra thin targets driven by high contrast laser pulses" Appl. Phys. Lett. 89, 2, 021502, 2006

$14 \mathrm{~S}$. Amoruso et al,"Characterisation of laser ablation of solid targets with near-infrarad laser pulses of 100 fs and 1 ps duration" Applied Surface Science, 252, p4863-4870, 2006.

15 D. Carroll, et al, "Dynamic control and enhancement of Laser accelerated protons" Comp. Rendus Phys. 10, 2-3, p188-196, 2009

$16 \mathrm{P}$. McKenna et al, "Effects of front surface plasma expansion on proton acceleration in ultraintense laser irradiation of foil targets" Laser and Particle Beams, 26, 4, p591-596, 2008

$17 \mathrm{~J}$. Andrews et al, "Transmission and laser damage studies of fused silica and PMMA debris shields with picosecond light pulse" SPIE 5647, p322-332, 2005

$18 \mathrm{C}$. Hernandez-Gomez et al, "Vulcan petawatt-operation and development" J. Phys. IV France 133, 555-559, 2006.

19 C. Ziener et al, "Specular reflectivity of plasma mirrors as a function of intensity, pulse duration and angle of incidence" J. Appl. Phys. 93, 1, 2003

$20 \mathrm{M}$. Streeter et al, "Relativistic plasma surfaces as an efficient second harmonic generators" New J. Phys. 13 023041, 2011

21 A. Pirozhkov et al, "Diagnostics of laser contrast using target reflectivity" Appl. Phys. Lett 94, 24, 241102, 2009

$22 \mathrm{C}$. Lin et al, "Enhancment of Ablation Efficiency by Femto/Nanosecond Dual-beam Micromachining System" Laser-based microand nanopackaging and assembly IV. SPIE 7585, 758501, 2010

$23 \mathrm{~F}$ Theberge et al, "Enhanced ablation of silica by superposition of fs and ns laser pulses", Appl. Phys. A80, 1505-10, 2005 